\title{
Search for methane isotope fractionation due to Rayleigh distillation on Titan
}

\author{
Máté Ádámkovics ${ }^{\mathrm{a}}$ and Jonathan L. Mitchell ${ }^{\mathrm{b}, \mathrm{c}}$ \\ ${ }^{a}$ Astronomy Department, University of California, Berkeley, CA 94720, USA \\ ${ }^{b}$ Department of Earth, Planetary and Space Sciences, \\ University of California, Los Angeles, CA 90095, USA \\ ${ }^{c}$ Department of Atmospheric and Oceanic Sciences, \\ University of California, Los Angeles, CA 90095, USA
}

\begin{abstract}
We search for meridional variation in the abundance of $\mathrm{CH}_{3} \mathrm{D}$ relative to $\mathrm{CH}_{4}$ on Titan using near-IR spectra obtained with NIRSPAO at Keck, which have a photon-limited signal-to-noise ratio of $\sim 50$. Our observations can rule out a larger than $10 \%$ variation in the column of $\mathrm{CH}_{3} \mathrm{D}$ below $50 \mathrm{~km}$. The preferential condensation of the heavy isotopologues will fractionate methane by reducing $\mathrm{CH}_{3} \mathrm{D}$ in the remaining vapor, and therefore these observations place limits on the amount of condensation that occurs in the troposphere. While previous estimates of $\mathrm{CH}_{3} \mathrm{D}$ fractionation on Titan have estimated an upper limit of $-6 \%$, assuming a solid condensate, we consider more recent laboratory data for the equilibrium fractionation over liquid methane, and use a Rayleigh distillation model to calculate fractionation in an ascending parcel of air that is following a moist adiabat. We find that deep, precipitating convection can enhance the fractionation of the remaining methane vapor by -10 to $-40 \%$, depending on the final temperature of the rising parcel. By relating fractionation of our reference parcel model to the pressure level where the moist adiabat achieves the required temperature, we argue that the measured methane fractionation constrains the outflow level for a deep convective event. Observations with a factor of at least 4 to 6 times larger signal-to-noise are required to detect this amount of fractionation, depending on the altitude range over which the outflow from deep convection occurs.
\end{abstract}

Keywords: Titan, atmosphere, Adaptive optics, Atmospheres, evolution, Atmospheres, structure

\section{Introduction}

Titan's hydrological cycle is an interesting combination of atmospheric and surface processes, and it is important for understanding the climate; for example, see the review by Mitchell and Lora (2016). Unlike on Earth, where the water of the hydro-

logical cycle is primarily in the condensed phase on the surface, Titan's supply of methane is stored mostly in the atmosphere as vapor (Lorenz et al., 2008). The column of methane vapor is equivalent to $5 \mathrm{~m}$ of liquid at the surface (Tokano et al., 2006). The large atmospheric reservoir contributes to the fact that the phase transitions of methane are a significant part of the energy transport in the atmosphere (Mitchell et al., 2009). The evaporation, circulation, and condensation of atmospheric methane redistributes the energy that is input as short wavelength solar radiation near to the equator, and moves it such that the outgoing long wavelength radiation at the top of the atmosphere is emitted nearly isotropically (Mitchell, 2012).

Condensation and evaporation are critical for determining which regions of the surface are dry or wet, with net evaporation drying the equatorial regions (Mitchell, 2008, Lora et al. 2015). While axisymmetric models of circulation predict the preponderance of ponding near the poles, they do not explain why the northern polar surface has significantly more and larger

Email addresses: mate@berkeley.edu (Máté Ádámkovics), jonmitch@g.ucla.edu (Jonathan L. Mitchell) lakes than in the south (Stofan et al., 2007; Hayes et al., 2008, Turtle et al. 2009). One explanation for the asymmetric distribution of polar lakes on Titan is Saturn's orbital eccentricity, which is thought to drive the seasonally-averaged preferential evaporation from the south (Aharonson et al., 2009). Tropospheric eddies, which models suggest are more vigorous in the south, are important for this process, as they pump the moisture that is evaporating from the surface up to higher altitudes and towards lower latitudes, and supply vapor to the upper atmosphere where the meridional overturning circulation takes place (Lora and Mitchell, 2015). Measuring the condensation of methane in the atmosphere, and how it changes with time, is one way to evaluate whether this explanation of the distribution of lakes is correct.

Indications of precipitation (Perron et al., 2006, Tokano et al. 2006; Ádámkovics et al. 2007, 2009; Mitchell et al., 2011; Turtle et al., 2011) and the observations of clouds (Brown et al. 2002; Roe et al. 2002; Roe, 2012) demonstrate that condensation is occuring in the atmosphere, but measurements of methane vapor are challenging. The Cassini-Huygens probe gas chromatograph mass spectrometer (GCMS) is the canonical measurement of methane (Niemann et al. 2010), and is often used as the point of reference for measurements at other locations and at other times. Cassini/CIRS spectra in the thermal IR are sensitive to both the methane abundance and temperature in stratosphere, and the mole fraction measured by the GCMS is used to retrieve thermal profiles (e.g., Flasar et al. 
2005: Achterberg et al. 2011). Lellouch et al. (2014) suggest that CIRS spectra can be used to measure the stratospheric methane abundance, since the mid- and far-IR lines of methane have different sensitivities to the thermal profile, and they find mole fractions near $\sim 85 \mathrm{~km}$ altitude of $\sim 1.0 \%$ at low latitudes, contrary to the GCMS. At $70 \mathrm{~N}$, where using the CIRS lines to constrain the temperature is challenging, Lellouch et al. (2014) find a mole fraction of $\sim 1.0 \%$, whereas Anderson et al. (2014) interpret the same observations with a temperature profile that was independently determined with Cassini Radio Science Subsystem (RSS) and find a mole fraction of $\sim 1.5 \%$. Near-IR measurements made with the Upward Looking Infrared Spectrometer (ULIS) of the Descent Imager/Spectral Radiometer (DISR) are consistent with the GCMS, and it has been suggested that the discrepancy with CIRS is due to uncertainties in methane line parameters (Bézard, 2014). On the other hand, the near-IR measurements of methane abundance are sensitive to properties of the atmospheric aerosol (Penteado et al. 2010, Ádámkovics et al., 2016). A method of measuring condensation that does not necessarily rely on the mole fraction would be a valuable complement to these techniques.

Condensation and evaporation impart an isotopic signature on a system because the rates of chemical and physical processes are different among isotopologues, due to the differences in mass, bonding, and zero point energies. In a closed system at equilibrium, the vapor pressures of different isotopologues are a measure of the difference in these rates. For a physical system like a parcel of air, where the condensate can be removed from the system, the fractionation can be larger still, as the fractionated condensate leaves the system. Rayleigh fractionation is therefore a signature of the magnitude and temperature at which evaporation and condensation occur, and measurements of ${ }^{18} \mathrm{O}$ and $\mathrm{D}$ in water are commonly used in interpreting properties of Earth's hydrological cycles (Dansgaard, 1964). Isotopic fractionation of condensible liquids is of considerable interest not only for characterizing the terrestrial water cycle (e.g., see review by $\mathrm{Xi}$, 2014), but also for constraining the Martian water cycle (e.g. Montmessin et al., 2005, Villanueva et al., 2015; Encrenaz et al., 2016). Similarly, the hydrological cycle on Titan can be informed by measurement of the isotopic composition of methane.

Recent measurements of the methane humidity in the lower atmosphere were made using spatially-resolved spectra in the $1.5 \mu \mathrm{m}$ (H-band) spectral region (Ádámkovics et al., 2016). Here we present further study of these spectra in the Section 2 , focusing on a detailed uncertainty analysis. The H-band is sensitive to two isotopologues of methane, offering the possibility of detecting variation in the strength of $\mathrm{CH}_{3} \mathrm{D}$ spectral features relative to those of $\mathrm{CH}_{4}$. While systematic instrumental noise and uncertainties in the gas-phase opacity are important, searching for variation in $\mathrm{CH}_{3} \mathrm{D}$ relative to $\mathrm{CH}_{4}$, and in spectra at one location relative to another, means that we can take into account the systematic effects. In Section 3 we describe the $S / N$ required to measure a given magnitude of fractionation, and in Section 4 we discuss these results interpreted with a Rayleigh fractionation model, where we estimate the magnitude of fractionation in a parcel of air with condensation.

\section{Methods}

\subsection{Observations}

The Near-InfraRed SPECctrometer (McLean et al., 1998) with adaptive optics (NIRSPAO) was used at W. M. Keck Observatory on 17 July 2014 UT to observe Titan with a spectral resolving power of $R \approx 25,000$ and a spatial sampling of $0.018 \%$ pixel along the slit. A single North-to-South position along the central meridian was integrated for $45 \mathrm{~min}$. We analyze spectra from one echelle order centered near $1.55 \mu \mathrm{m}$. Additional details of these observations, including the data reduction and calibration with supporting datasets, are described in Ádámkovics et al. (2016).

\subsection{Radiative Transfer Model}

Synthetic spectra are generated by defining 20 atmospheric layers, with properties that are determined primarily by measurements made with instruments on the Huygens probe. The layers have boundaries (levels) that are evenly spaced in pressure, with 10 levels above and 10 levels below 300 mbar (see Table 2 in Ádámkovics et al., 2016). The top of the atmosphere is set at zero optical depth. We use measurements of the temperature, pressure, methane abundance, and aerosol structure to determine the gas and scattering opacity in each layer. The methane below $35 \mathrm{~km}$ and aerosol above $65 \mathrm{~km}$ are assumed to vary with latitude, as detailed in Ádámkovics et al. (2016). Methane is increasing linearly from $40 \mathrm{~N}$ toward the south, reaching a $30 \%$ enhancement at $40 \mathrm{~S}$, while the aerosol is increasing in opacity toward the north. The Huygens temperature profile is used at all latitudes. $\mathrm{CH}_{4}$ and $\mathrm{CH}_{3} \mathrm{D}$ line opacities are from the HITRAN 2012 database (Rothman et al., 2013). The discrete-ordinate-method radiative transfer(DISORT; Stamnes et al., 1988) is implemented in Python (PyDISORT) and used to solve the radiative transfer through the model atmosphere and simulate the observed flux.

Each $(x, y)$ pixel on the detector maps to a wavelength and latitude $\left(\lambda_{x}, \phi_{y}\right)$ for the observed flux, $I_{\text {obs }}\left(\lambda_{x}, \phi_{y}\right)$. We use the radiative transfer model to calculate the flux $I_{\text {calc }}\left(\lambda_{x}, \phi_{y}\right)$ corresponding to this wavelength and location, taking into account the viewing geometry and spatial variation in surface albedo and hazes (Ádámkovics et al. 2016). An example spectrum from one NIRSPAO pixel along the slit (out of a total of 44 that cover the disk of Titan), is compared with the radiative transfer model calculation in Figure 1 .

\subsection{Uncertainty Analysis}

By inspecting a 300s exposure, we find the background count rate at echelle Order 49 to be $0.21 \mathrm{DN} / \mathrm{s}$, while the count rate on Titan ranges from 0.08 to $0.63 \mathrm{DN} / \mathrm{s}$, in the dark and bright spectral regions, respectively. Using a NIRSPAO dark current of $0.7 \mathrm{e}^{-} / \mathrm{s} /$ pix, gain of $5.7 \mathrm{e}^{-} / \mathrm{DN}$, read noise of $23 \mathrm{e}^{-}$, and total $9 \times 300$ s exposure time, the counting-limited signal-to-noise ratio $\mathrm{S} / \mathrm{N}$ per pixel ranges from 19 in the dark regions to 87 where Titan is bright. We'll use the mean value to characterize the entire spectrum as $\mathrm{S} / \mathrm{N} \approx 50$. In units of reflectivity, which cover a range of roughly 0.03 to $0.12 I / F$, this level of noise 


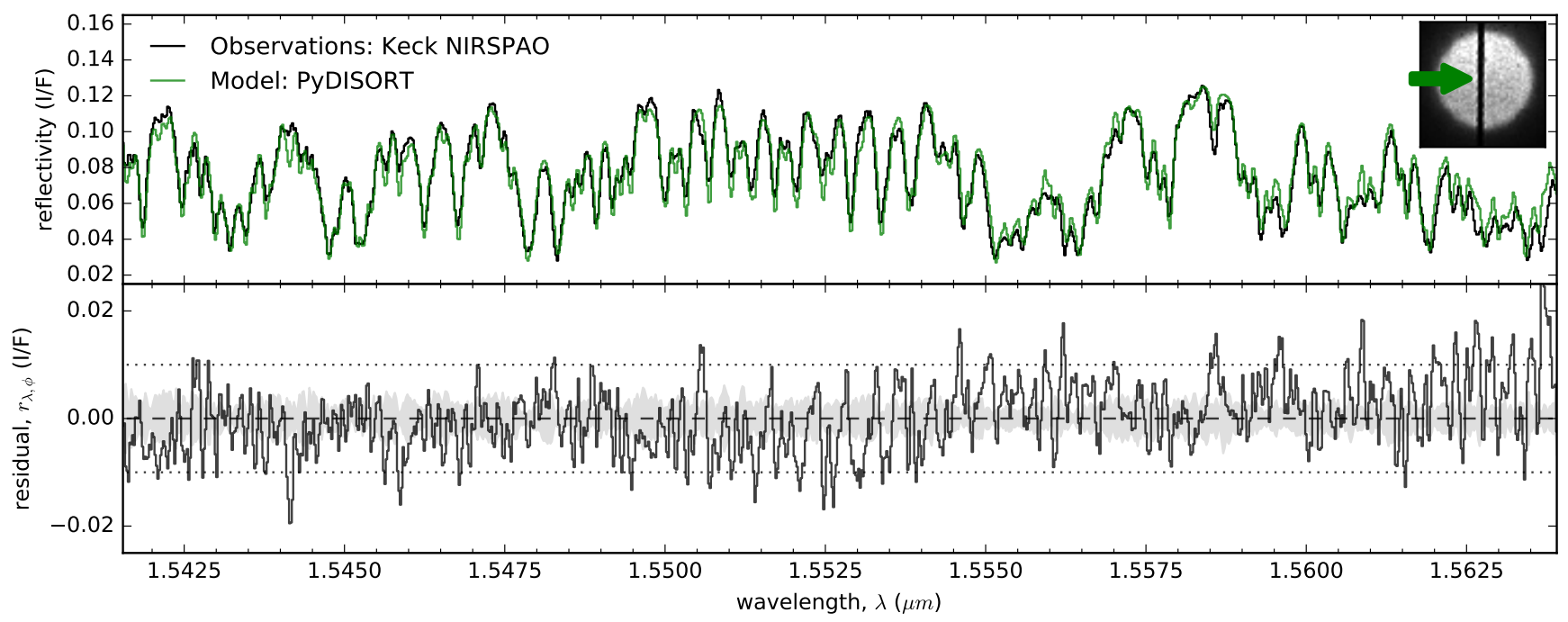

Figure 1: The spectrum from one NIRSPAO pixel (top panel; black), at the location specified by the green arrow in the slit-viewing camera image (inset), is compared with the radiative transfer model spectrum (green). The residuals are plotted in the bottom panel, with the ordinate scale magnified by a factor of three relative to the top panel. The shaded grey region is the standard deviation of the observed flux from the neighboring five pixels, which gives an estimate of the pixel-to-pixel noise in the observations.
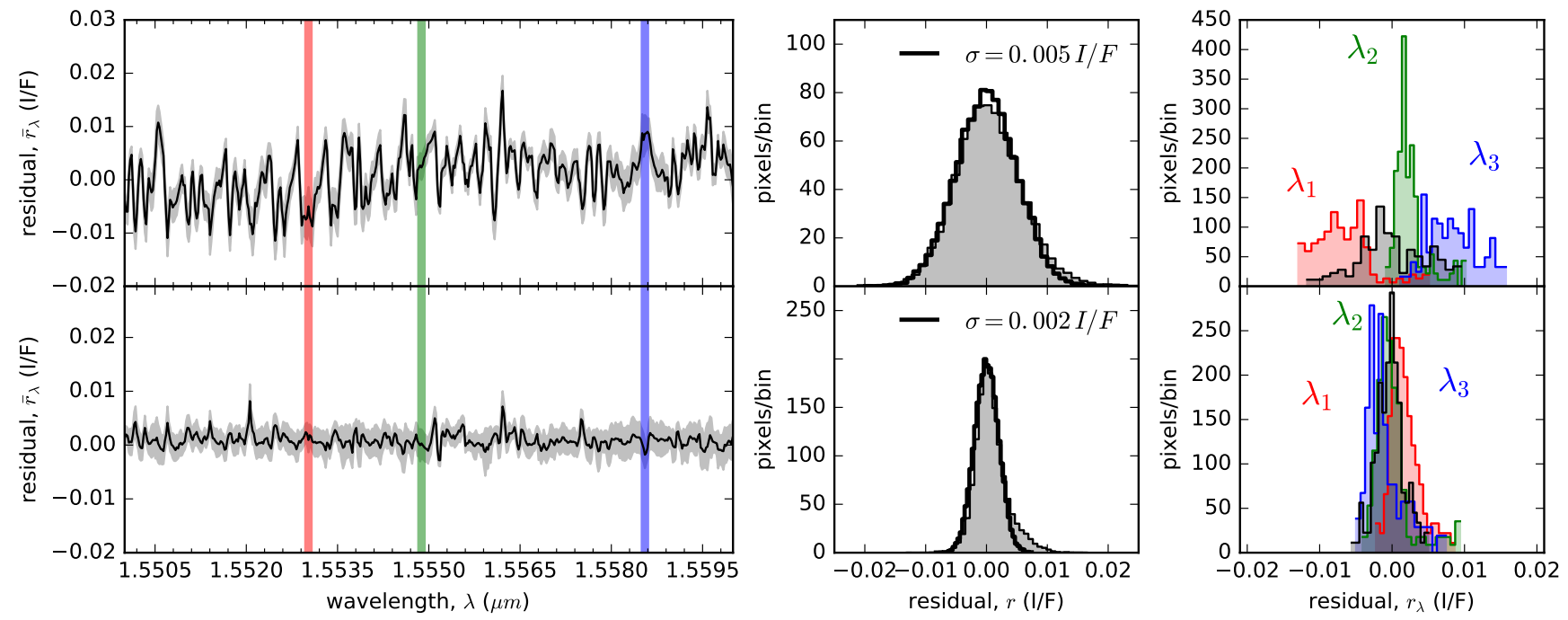

Figure 2: Residuals for the standard model (top row of panels) are chacterized by variations in $\bar{r}_{\lambda}$ (black line) that are larger than $\sigma_{\lambda}$ (grey shaded region). The center panel is a histogram of the residuals $r$ for the entire sample (grey) shown with a histogram for a random normal distribution of the same sample size with $\sigma=0.005$. Residuals from wavelengths corresponding to the vertical lines of matching color are plotted in the right panel, which can be discrepant from a histogram of a random noise (black). The bottom row of panels is for residuals from models that include an empirical correction for the systematic error (see text for details). After correction, the structure in the spectrum of $\bar{r}_{\lambda}$ is removed (left panel), $r$ is consistent with random noise with $\sigma=0.002$ (center panel), and $r_{\lambda}$ is distributed around zero for individual wavelengths (right panel). 
corresponds to $\sim 0.0015 I / F$ per pixel and sets the limit for the expected residuals when comparing these observations to our models.

The residual for each pixel is defined with the following notation,

$$
r_{\lambda, \phi}=I_{\mathrm{obs}}\left(\lambda_{x}, \phi_{y}\right)-I_{\mathrm{calc}}\left(\lambda_{x}, \phi_{y}\right),
$$

and we consider the standard deviation $\sigma$ for the entire sample of residuals $r$, as well as sub-samples at a specific wavelength $r_{\lambda}$ or latitude $r_{\phi}$, which have mean residuals and standard deviations of $\bar{r}_{\lambda}, \bar{r}_{\phi}$ and $\sigma_{\lambda}, \sigma_{\phi}$, respectively.

The residuals that are shown in Figure 1 are often in excess of the per pixel noise of the observations determined above, as well as the pixel-to-pixel noise of the observations determined by comparing nearby pixels. This indicates that systematic errors dominate and is supported by the similarity of the residuals at other spatial locations (see, e.g,. Figure 5 in Ádámkovics et al. (2016)). The systematic error is illustrated with a spectrum of the mean residual for all spatial pixels, $\bar{r}_{\lambda}$, in Figure 2 . For comparison, we generate a random normal sample of Gaussian noise in a two-dimensional array that is the same size as the observations and compare with the residuals. The entire sample $r$ is characterized by a normal distribution with $\sigma=0.005 I / F$, in excess of the estimated per pixel noise of the observations. The distribution of residuals at individual wavelengths are different, and inconsistent with random noise. At wavelengths where the systematic error is greatest, $\left|\bar{r}_{\lambda}\right|>\sigma_{\lambda}$, indicating significant discrepancy.

The systematic spectral error could arise from some combination of the following: either inadequate removal of contamination (e.g., from scattered light) or other systematic instrumental noise during the data reduction, or uncertainties in the gas phase opacities considered in the radiative transfer model. The latter could involve either ignoring gas phase species that contribute to the spectrum, or limitations in the absorption coefficients. Since the features in the spectrum of $\bar{r}_{\lambda}$ have widths that are comparable to the methane spectrum, the systematic error may have a contribution from limitations in the gas opacity.

To compensate for the systematic error, we implement an empirical correction to the gas opacity. Since methane dominates the gas opacity, we make an ad hoc correction to the methane opacities. The corrected methane opacity spectrum $\tau_{\text {corr }}(\lambda)$ is calculated using the standard methane opacity $\tau(\lambda)$ and residuals $\bar{r}_{\lambda}$,

$$
\tau_{\mathrm{corr}}(\lambda)=\tau(\lambda) \exp \left(c_{0} \bar{r}_{\lambda}\right)
$$

where the coefficient $c_{0}$ is set to minimize the residuals in the models using the corrected opacities (in this case $c_{0}=60$ ). The calculation of $\tau(\lambda)$ is described in Ádámkovics et al. (2016). The same correction factor, $\exp \left(c_{0} \bar{r}_{\lambda}\right)$, is applied to all layers in the model and to all latitudes. Residuals from a model that used these corrected opacities are shown in the bottom row of Figure 2, with $\sigma=0.002 \mathrm{I} / \mathrm{F}$, which is close to the photonlimited per pixel $\mathrm{S} / \mathrm{N}$ of $0.0015 I / F$. A tail in the distribution of $r$ to large positive values (and spikes in the spectrum of $\bar{r}_{\lambda}$ ) still exist. This ad hoc correction to the model consolidates all

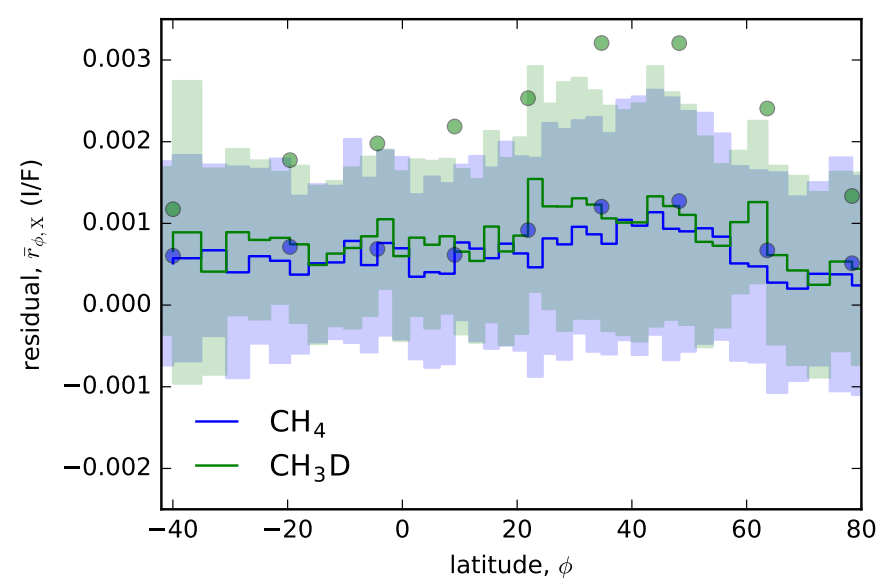

Figure 4: Meridional trends in the median residual at wavelengths that are preferentially sensitive to $\mathrm{CH}_{3} \mathrm{D}$ (green) and $\mathrm{CH}_{4}$ (blue). The shaded regions are the uncertainty $\sigma_{\phi, X}$ for the sample at each latitude. Residuals in a model where $\mathrm{CH}_{3} \mathrm{D}$ is decreased by $10 \%$ are shown as circles, indicating that a reduction of this magnitude is detectable when considering these wavelengths.

of the systematic uncertainty into the methane opacity, and because there are correction factors of up to a factor of 2 , it is unlikely that there are inaccuracies of this magnitude in the laboratory data. If future observations show a similar spectrum of systematic noise, especially if the instrument grating and crossdisperser angles are different, resulting in the spectra falling on different pixels, then it may suggest that the methane opacities could indeed be the culprit; however, if the systematic noise is different, it will point to an instrumental artifact.

\section{Results}

There are several wavelength regions (or channels) near $1.5 \mu \mathrm{m}$ that are sensitive to $\mathrm{CH}_{3} \mathrm{D}$. Transmission spectra for both isotopologues are shown in the top panel of Figure 3 and the wavelength regions where the $\mathrm{CH}_{3} \mathrm{D}$ opacity exceeds that of $\mathrm{CH}_{4}$ by at least $15 \%$ are highlighted (103 channels altogether). Collectively, this sample of wavelengths covers a range of $I / F$, and are therefore sensitive to $\mathrm{CH}_{3} \mathrm{D}$ at various altitudes in the atmosphere. We randomly sample 103 channels from the remaining 900 that are sensitive to $\mathrm{CH}_{4}$ for a baseline comparison. The meridional trend in the mean of the residuals for these two sets of channels $\bar{r}_{\phi, \mathrm{X}}$, where $\mathrm{X}$ specifies $\mathrm{CH}_{3} \mathrm{D}$ or $\mathrm{CH}_{4}$, are plotted in Figure 4, showing that no variation in $\mathrm{CH}_{3} \mathrm{D}$ relative to $\mathrm{CH}_{4}$ is detected.

To determine what our sensitivity is with a per pixel noise of $0.002 \mathrm{I} / \mathrm{F}$, we first consider a $10 \%$ decrease in $\mathrm{CH}_{3} \mathrm{D}$ relative to $\mathrm{CH}_{4}$ below $50 \mathrm{~km}$ altitude. The datapoints in Figure 4 illustrate that decreasing the abundance of $\mathrm{CH}_{3} \mathrm{D}$ by $10 \%$, while keeping $\mathrm{CH}_{4}$ fixed, causes a model residual outside the uncertainty over a significant fraction of the disk. There is increased sensitivity near and sub-observer point where the $10 \%$ decrease causes the larger residuals that occur near the limb. There are also large residuals toward Northern latitudes where the surface albedo is 


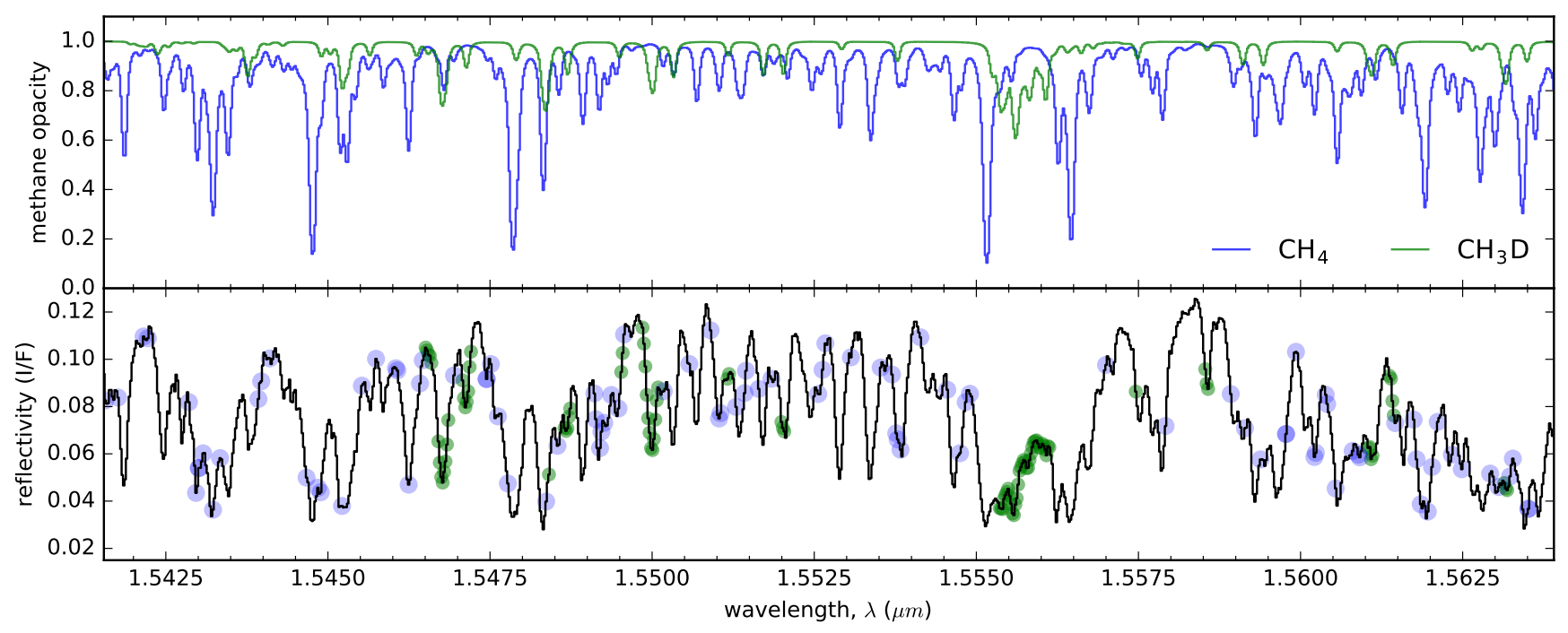

Figure 3: Transmission spectra of $\mathrm{CH}_{4}$ (blue) and $\mathrm{CH}_{3} \mathrm{D}$ (green) in the top panel at a temperature $T=94 \mathrm{~K}$, pressure $P=1$ bar, and unit path length. The wavelength regions where $\mathrm{CH}_{3} \mathrm{D}$ dominates the methane gas opacity are highlighted with green circles in the observed spectrum in the bottom panel. A random selection of the same number of datapoints that are sensitive to $\mathrm{CH}_{4}$ are shown in blue. These two sets of wavelengths are used to search for spatial variation in $\mathrm{CH}_{3} \mathrm{D}$ relative to $\mathrm{CH}_{4}$.

higher and the returned flux is greater. As a comparison, the set of baseline wavelengths, which are more sensitive to $\mathrm{CH}_{4}$, show little variation in the residuals when only the abundance of $\mathrm{CH}_{3} \mathrm{D}$ is changed. The systematic error is a factor of 2.5 larger for the entire sample (see Figure 2). If the empirical correction is not included, this corresponds to a sensitivity to a $25 \%$ decrease in $\mathrm{CH}_{3} \mathrm{D}$.

To generalize the sensitivity to changes in $\mathrm{CH}_{3} \mathrm{D}$ abundance for a particular $\mathrm{S} / \mathrm{N}$, we compare models with a range of $\mathrm{CH}_{3} \mathrm{D}$ abundances to our reference model. We use the standard notation for isotopic variation,

$$
\delta=\left(a-a_{\text {std }}\right) / a_{\text {std }} \times 10^{3} \%
$$

where the absolute content $a$ of an isotope is compared to a standard reference value $a_{\text {std }}$. For the two isotopologues of methane we consider specifically

$$
\delta \mathrm{CH}_{3} \mathrm{D}=\frac{\left(\frac{\mathrm{CH}_{3} \mathrm{D}}{\mathrm{CH}_{4}}\right)-\left(\frac{\mathrm{CH}_{3} \mathrm{D}}{\mathrm{CH}_{4}}\right)_{\text {std }}}{\left(\frac{\mathrm{CH}_{3} \mathrm{D}}{\mathrm{CH}_{4}}\right)_{\text {std }}} \times 10^{3} \% \text {, }
$$

where were use the terrestrial $\mathrm{D} / \mathrm{H}=1.56 \times 10^{-4}$ for our standard $\left(\mathrm{CH}_{3} \mathrm{D} / \mathrm{CH}_{4}\right)_{\text {std }}$, which is consistent with the isotope ratio observed on Titan (Nixon et al., 2012).

We calculate the mean residual $\bar{r}_{\delta}$, between the standard calculation and one with $\delta \mathrm{CH}_{3} \mathrm{D}$ depletion, over the sample of wavelengths that are shown to be sensitive to $\mathrm{CH}_{3} \mathrm{D}$ in Figures 3 and 4 . If the noise in the observations is $\mathrm{N}<\bar{r}_{\delta}$, the variation of $\delta \mathrm{CH}_{3} \mathrm{D}$ is detectable. By considering a characteristic signal of magnitude $\mathrm{S}=0.1 \mathrm{I} / \mathrm{F}$, we can then determine the limiting $\mathrm{S} / \mathrm{N}$ required to measure a particular depletion. A contour plot of the limiting $\mathrm{S} / \mathrm{N}$ required for these two parameters is shown in Figure 5. Depletion from below lower altitudes

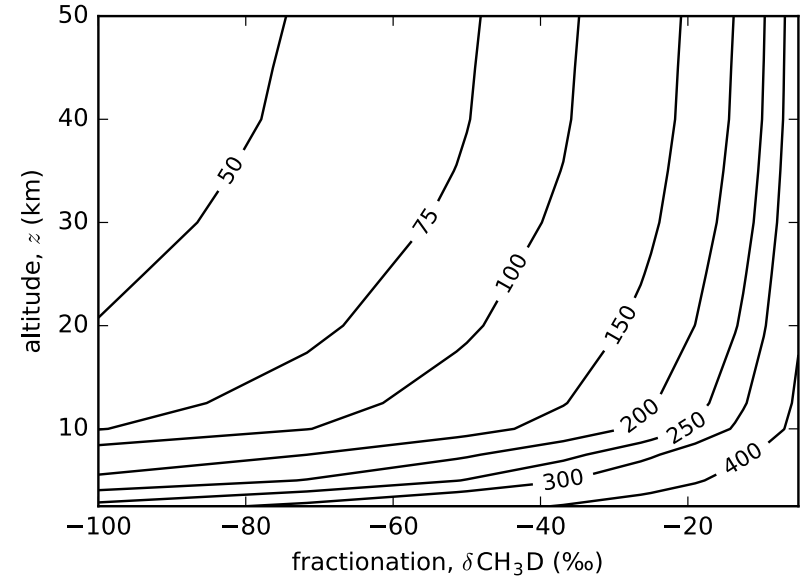

Figure 5: The per pixel S/N that is required to observe a $\delta \mathrm{CH}_{3} \mathrm{D}$ of fractionation below a given altitude $z$ in the atmosphere.

means that a smaller column of $\mathrm{CH}_{3} \mathrm{D}$ is removed, so that the resulting change in the spectrum is smaller, requiring a higher $\mathrm{S} / \mathrm{N}$ to detect. Similarly, smaller depletions at a given altitude require higher $\mathrm{S} / \mathrm{N}$.

\section{Discussion}

If condensation occurs under Rayleigh conditions, where the condensate is immediately removed from the vapor after formation, and we assume that process is isothermal at temperature $T$, 


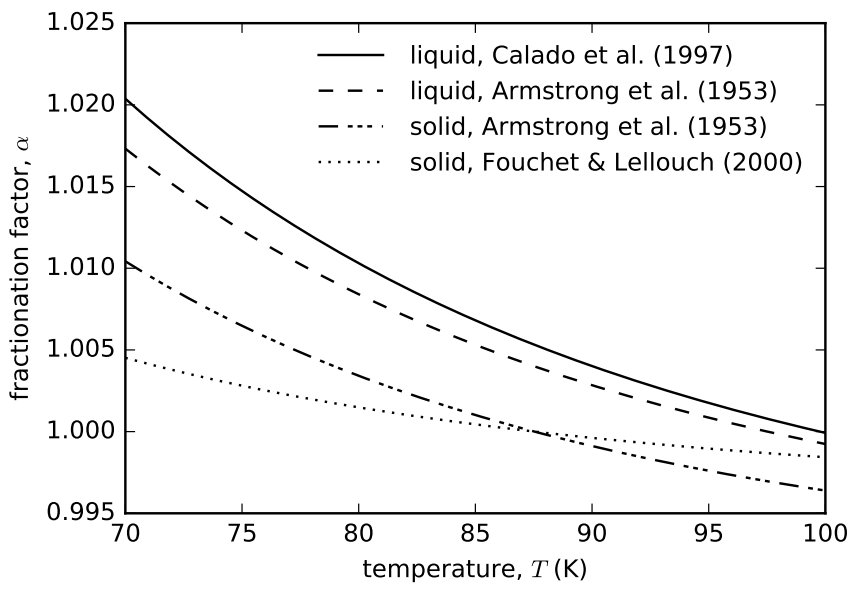

Figure 6: The fractionation factor, $\alpha$, for vapor in equilibrium with solid and liquid methane from the two-parameter fits to the laboratory data of Armstrong et al. (1953) and Calado et al. (1997), along with a curve for the expression used by Fouchet and Lellouch (2000).

then the isotopic fractionation for the vapor and condensate are

$$
\delta_{v}=\frac{1}{\alpha} F_{v}^{\alpha-1}-1
$$

and

$$
\delta_{c}=F_{v}^{\alpha-1}-1
$$

respectively, where $\alpha$ is the fractionation factor (or fractionation coefficient) and $F_{v}$ is the remaining fraction of vapor (Dansgaard, 1964). The fractionation factor is the ratio of vapor pressures for the two isotopologues.

Laboratory measurements of the vapor pressures of methane isotopologues in equilibrium with solid and liquid methane at different temperatures are reported with various two-parameters fits (Armstrong et al., 1953, Calado et al., 1997), and are shown in Figure 6 Also included is the expression used by Fouchet and Lellouch (2000), where they assumed a maximum $\alpha=1.004$ at $71 \mathrm{~K}$, which they used to calculate a maximum $-6 \%$ fractionation on Titan for solid methane condensate.

Here we use the more recent laboratory measurements from Calado et al. (1997) for the liquid methane fractionation factor for $\mathrm{CH}_{3} \mathrm{D} / \mathrm{CH}_{4}$,

$$
\alpha=\exp \left(\frac{A}{T^{2}}-\frac{B}{T}\right)
$$

with $A=331.29 \mathrm{~K}^{2}$ and $B=3.3208 \mathrm{~K}$. At $85 \mathrm{~K}, \alpha=1.0068$, and we can use Equations 5 and 6 to calculate the $\mathrm{CH}_{3} \mathrm{D}$ depletion in the vapor that is expected during methane condensation in a parcel where precipitation removes the liquid condensate. Curves for the expected fractionation of the condensate and vapor during condensation are shown in Figure 7 . Since we make the simplifying assumption that condensation is isothermal, we also calculate $\delta \mathrm{CH}_{3} \mathrm{D}$ at $80 \mathrm{~K}$ and $90 \mathrm{~K}$ (left panel of Figure 8). These temperatures apply to the lower troposphere, up to an altitude of $\sim 15 \mathrm{~km}$. Coincidentally, a fractionation factor of

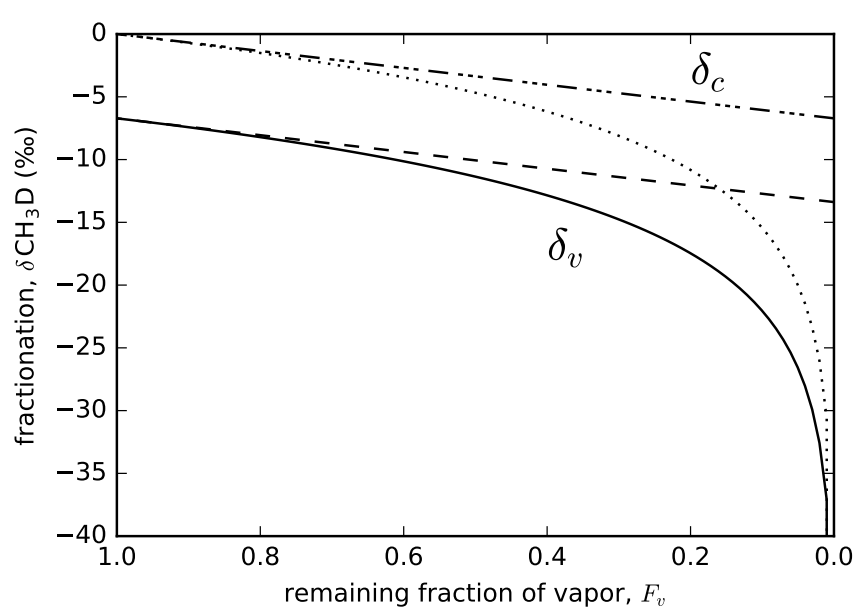

Figure 7: The fractionation of methane in the remaining vapor $\delta_{v}$ and in the condensate $\delta_{c}$, during condensation using an equilibrium model (dashed lines) and isothermal Rayleigh distillition at $85 \mathrm{~K}$ (solid and dotted curves), where the condensate is removed from the system.

$\alpha \approx 1.010$ for vapor over liquid methane at $80 \mathrm{~K}$ is roughly the same as for vapor over solid methane at $70 \mathrm{~K}$, which would be applicable at higher altitudes, in the $30-50 \mathrm{~km}$ range. Predictions for fractionation based on an isothermal Rayleigh distillation model fall in the -5 to $-40 \%$ range, depending on temperature and the fraction of vapor that condenses. While these estimates bracket a range of plausible values, we next consider a more realistic scenario.

Our working hypothesis is that methane vapor is supplied from near-surface environments to the upper troposphere by localized, deep (precipitating) convection, such as the storms observed at the south pole following the southern-hemisphere summer solstice (e.g., Schaller et al., 2006a b) and near the equator at equinox (Turtle et al., 2011). We imagine a surfacelevel parcel of air that is lifted beyond the condensation level following the moist adiabat. The parcel therefore maintains a saturated value of humidity, losing the excess to condensation of cloud droplets/ice and precipitation. In a vigorous updraft, the parcel reaches a stable, equilibrium level and is forced to outflow laterally into the surrounding environment. Because Titan has such weak lateral temperature gradients, the parcel's humidity upon outflow is conserved. Therefore the parcel retains the isotope signal it inherited at the point of outflow from the convective updraft. Because Huygens observed nearly saturated methane vapor above the boundary layer, it seems plausible that many such parcels have mixed to essentially saturate the entire troposphere above the boundary layer $(\sim 5-40 \mathrm{~km}$ altitude) with methane.

With an estimate of the temperature and dew point temperature (or any other measure of methane vapor concentration) of a near-surface parcel, our reference model of how methane vapor is supplied to Titan's free atmosphere (all levels above the boundary layer) provides all the necessary ingredients to relate 

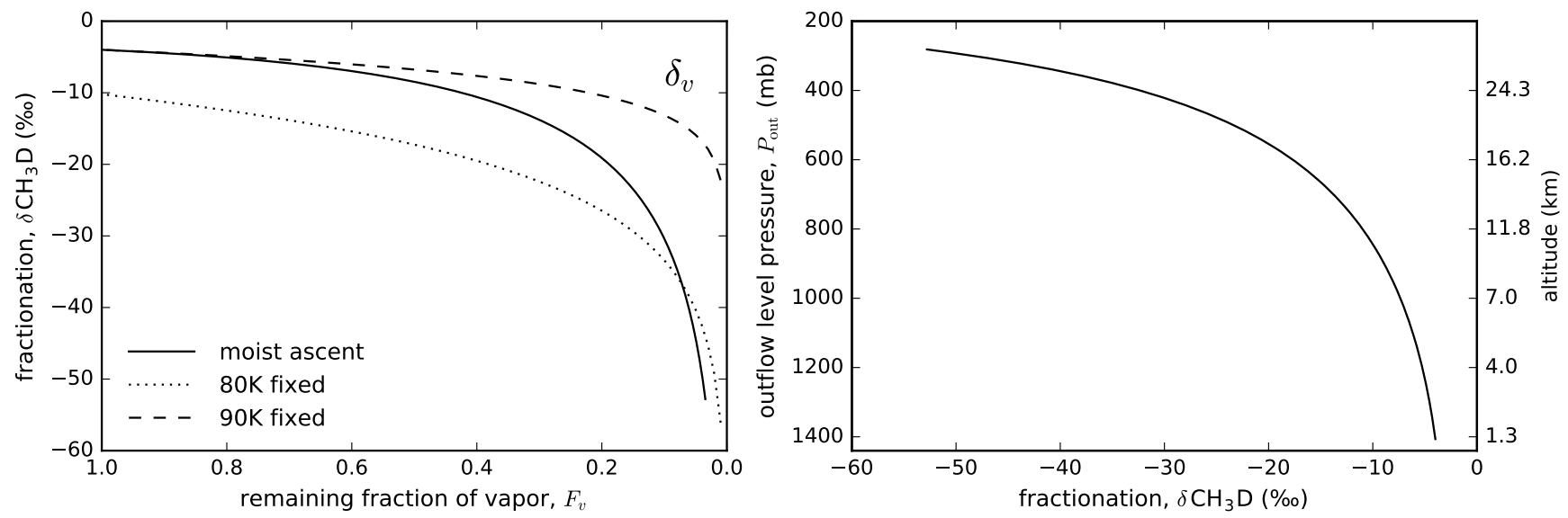

Figure 8: The methane vapor fractionation for a parcel of air ascending along the moist adiabat from the LCL (solid curve) is compared with isothermal fractionation at $80 \mathrm{~K}$ (dotted curve) and $90 \mathrm{~K}$ (dashed) in the left panel. Since the temperature and remaining fraction of vapor are known for a parcel ascending along the moist adiabat, the fractionation in the vapor can be used to determine the outflow pressure level for a precipitating deep convective event (right panel).

changes in vapor fractionation with changes in outflow levels. Here we provide a sample calculation to illustrate the magnitude of fractionation changes that could be present in Titan's methane vapor. We start with the idealized Clausius-Clapeyron equation

$$
e_{s}(T)=e_{s_{0}} \exp \left[\frac{H_{l v}}{R_{v}}\left(\frac{1}{T_{0}}-\frac{1}{T}\right)\right]
$$

with triple-point pressure, $e_{s_{0}}$, and temperature, $T_{0}$, latent heat of vaporization, $H_{l v}$ and methane gas constant, $R_{v}$. Alternative vapor pressure curves could be implemented, however the magnitude of the uncertainty due to the choice of an idealized expression is negligible relative to other uncertainties, such as the isotopic composition of the methane source region. The lifting condensation level (LCL) can be found by following a dry adiabat up from the surface parcel until the dew point temperature is reached. Above this level, we assume the parcel maintains saturation by condensing/freezing the excess and immediately removing the liquid/solid portion from the parcel. The parcel fractionation at any level is the sum of all the incremental condensation that occurs as the parcel is lifted to that level. In other words, we must integrate,

$$
\frac{d \delta_{v}}{d T}=\frac{F_{v}^{\alpha-2}}{\alpha^{2}}\left[F_{v} \frac{d \alpha}{d T}\left(\alpha \ln F_{v}-1\right)+(\alpha-1) \alpha \frac{d F_{v}}{d T}\right],
$$

over the temperature range experienced by the parcel during ascent along the moist adiabat. Equation 9 is found by differentiating the equation for $\delta_{v}(T)$, Equation 5, with respect to temperature.

The left panel of Figure 8 displays the results of this integration for a parcel with a $90 \mathrm{~K}$ dew point temperature starting at an LCL of $\sim 1400$ mbar up to a level where the moist adiabat reaches $70 \mathrm{~K}$, and as a function of the fraction of remaining vapor. For comparison, the fractionation curves for isothermal parcels at $80 \mathrm{~K}$ and $90 \mathrm{~K}$ are also shown. Because the parcel's temperature is constrained to be on the moist adiabat, and because we know the fraction of remaining vapor at saturation given the temperature, we can use the moist adiabat, $T_{m a}(p)$, to map the value of $F_{v}$ onto the outflow level pressure, $P_{\text {out }}$, of the parcel; this is shown in the right panel of Figure 8 . From this figure we see that a deep, precipitating convective event with an outflow level of 400 mbar will have fractionated the source parcel's methane by $\sim 50$ per mil. Since we do not know the parcel's initial isotopic composition, we therefore do not necessarily know the correct $\mathrm{CH}_{3} \mathrm{D} / \mathrm{CH}_{4}$ standard against which to measure the fractionation, However, if large differences in methane vapor fractionation are observed in latitude or altitude, we may infer that outflow from deep convection occurs at varying levels. This is, in fact, expected to be the case; polar convection is likely to reach higher altitudes (lower pressures) than lowlatitude convection, presumably because surface parcels have higher humidities at high latitudes (Griffith et al., 2008).

Nixon et al. (2012) describe measurements of isotopic composition made with the CIRS instrument on the Cassini spacecraft, which are sensitive to stratospheric altitudes ( 80 $280 \mathrm{~km}$ ), finding that the $\mathrm{D} / \mathrm{H}$ ratios in methane are consistent with the terrestrial value. They also summarize previous measurements from the literature, including ground-based measurements in the near-IR that are sensitive to a methane column that extends down to the surface (Penteado et al., 2005; de Bergh et al. 2012). It may be interesting to note that while the groundbased measurements agree within uncertainties with the CIRS observations, they correspond to slightly smaller $\mathrm{D} / \mathrm{H}$ ratios than those measured in the stratosphere. Determining whether or not this is due to actual altitude or temporal variation in the isotopic composition, rather than systematic differences among the measurements, will require higher signal-to-noise observations with smaller measurement uncertainties.

Recent $a b$ initio calculations have been used to make additional rovibrational assignments of $\mathrm{CH}_{4}$ lines and sub-bands 
(Rey et al. 2016), which can be used to (re)evaluate the uncertainties in the methane opacities used in our model. While implementing and critically evaluating these recent assignments is beyond the scope of this work, future observations at higher $\mathrm{S} / \mathrm{N}$, together with future assignments of currently unassigned $\mathrm{CH}_{3} \mathrm{D}$ lines, will provide a means to reduce the systematic uncertainties in the analysis presented here.

In summary, we have revisited high resolution NIRSPAO observations of Titan searching for a spatial variation in the isotopic ratio of $\mathrm{D} / \mathrm{H}$ in methane. We can exclude variation in $\mathrm{CH}_{3} \mathrm{D}$ that is greater than $10 \%$ with the current observations. We describe isothermal models of Rayleigh distillation, which predict that fractionation of -10 to $-40 \%$ should occur for a condensing parcel of air, and we show that fractionation of this magnitude can be used to determine the outflow level for a deep convective event. We predict that NIRSPAO observations with a factor of 4 to 6 higher $\mathrm{S} / \mathrm{N}$ are required to measure depletions of this magnitude. Such observations will be facilitated by the NIRSPEC instrument upgrade (Martin et al. 2014), and will be feasible with the next generation of ground-based telescopes such as the Thirty Meter Telescope (Skidmore et al. 2015) and the European Extremely Large Telescope (Tamai and Spyromilio, 2014).

\section{Acknowledgements}

This work was supported by NASA grants NNX14AG82G and NNX12AM81G, and the manuscript was improved with the constructive comments of two anonymous reviewers.

\section{References}

\section{References}

Achterberg, R. K., Gierasch, P. J., Conrath, B. J., Michael Flasar, F., Nixon, C. A., Jan. 2011. Temporal variations of Titan's middle-atmospheric temperatures from 2004 to 2009 observed by Cassini/CIRS. Icarus 211, 686-698.

Ádámkovics, M., de Pater, I., Hartung, M., Barnes, J. W., Nov. 2009. Evidence for condensed-phase methane enhancement over Xanadu on Titan. Planet. Space Sci. 57, 1586-1595.

Ádámkovics, M., Mitchell, J. L., Hayes, A. G., Rojo, P. M., Corlies, P., Barnes, J. W., Ivanov, V. D., Brown, R. H., Baines, K. H., Buratti, B. J., Clark, R. N., Nicholson, P. D., Sotin, C., May 2016. Meridional variation in tropospheric methane on Titan observed with AO spectroscopy at Keck and VLT. Icarus 270, 376-388.

Ádámkovics, M., Wong, M. H., Laver, C., de Pater, I., Nov. 2007. Widespread Morning Drizzle on Titan. Science 318, 962-965.

Aharonson, O., Hayes, A. G., Lunine, J. I., Lorenz, R. D., Allison, M. D., Elachi, C., Dec. 2009. An asymmetric distribution of lakes on Titan as a possible consequence of orbital forcing. Nature Geoscience 2, 851-854.

Anderson, C. M., Samuelson, R. E., Achterberg, R. K., Barnes, J. W., Flasar, F. M., Nov. 2014. Subsidence-induced methane clouds in Titan's winter polar stratosphere and upper troposphere. Icarus 243, 129-138.

Armstrong, G. T., Brickwedde, F. G., Scott, R. B., Jul. 1953. The Vapor Pressures of the Deuteromethanes. J. Chem. Phys. 21, 1297-1298.

Bézard, B., Nov. 2014. The methane mole fraction in Titan's stratosphere from DISR measurements during the Huygens probe's descent. Icarus 242, 64 73.

Brown, M. E., Bouchez, A. H., Griffith, C. A., Dec. 2002. Direct detection of variable tropospheric clouds near Titan's south pole. Nature 420, 795-797.

Calado, J. C. G., Lopes, J. N. C., Nunes da Ponte, M., Rebelo, L. P. N., Jun. 1997. Vapor pressure of partially deuterated methanes $\left(\mathrm{CH}_{3} \mathrm{D}, \mathrm{CH}_{2} \mathrm{D}_{2}\right.$, and $\mathrm{CHD}_{3}$ ). J. Chem. Phys. 106, 8792-8798.
Dansgaard, W., Nov. 1964. Stable isotopes in precipitation. Tellus 16, 436.

de Bergh, C., Courtin, R., Bézard, B., Coustenis, A., Lellouch, E., Hirtzig, M., Rannou, P., Drossart, P., Campargue, A., Kassi, S., Wang, L., Boudon, V., Nikitin, A., Tyuterev, V., Feb. 2012. Applications of a new set of methane line parameters to the modeling of Titan's spectrum in the $1.58 \mu \mathrm{m}$ window. Planet. Space Sci. 61, 85-98.

Encrenaz, T., DeWitt, C., Richter, M. J., Greathouse, T. K., Fouchet, T., Montmessin, F., Lefèvre, F., Forget, F., Bézard, B., Atreya, S. K., Case, M., Ryde, N., Feb. 2016. A map of D/H on Mars in the thermal infrared using EXES aboard SOFIA. A\&A 586, A62.

Flasar, F. M., Achterberg, R. K., Conrath, B. J., Gierasch, P. J., Kunde, V. G., Nixon, C. A., Bjoraker, G. L., Jennings, D. E., Romani, P. N., Simon-Miller, A. A., Bézard, B., Coustenis, A., Irwin, P. G. J., Teanby, N. A., Brasunas, J., Pearl, J. C., Segura, M. E., Carlson, R. C., Mamoutkine, A., Schinder, P. J., Barucci, A., Courtin, R., Fouchet, T., Gautier, D., Lellouch, E., Marten, A., Prangé, R., Vinatier, S., Strobel, D. F., Calcutt, S. B., Read, P. L., Taylor, F. W., Bowles, N., Samuelson, R. E., Orton, G. S., Spilker, L. J., Owen, T. C., Spencer, J. R., Showalter, M. R., Ferrari, C., Abbas, M. M., Raulin, F., Edgington, S., Ade, P., Wishnow, E. H., May 2005. Titan's Atmospheric Temperatures, Winds, and Composition. Science 308, 975-978.

Fouchet, T., Lellouch, E., Mar. 2000. Vapor Pressure Isotope Fractionation Effects in Planetary Atmospheres: Application to Deuterium. Icarus 144, 114 123.

Griffith, C. A., McKay, C. P., Ferri, F., Nov. 2008. Titan's Tropical Storms in an Evolving Atmosphere. Astrophys. J. Lett. 687, L41-L44.

Hayes, A., Aharonson, O., Callahan, P., Elachi, C., Gim, Y., Kirk, R., Lewis, K., Lopes, R., Lorenz, R., Lunine, J., Mitchell, K., Mitri, G., Stofan, E., Wall, S., May 2008. Hydrocarbon lakes on Titan: Distribution and interaction with a porous regolith. Geophysical Research Letters 35, L9204.

Lellouch, E., Bézard, B., Flasar, F. M., Vinatier, S., Achterberg, R., Nixon, C. A., Bjoraker, G. L., Gorius, N., Mar. 2014. The distribution of methane in Titan's stratosphere from Cassini/CIRS observations. Icarus 231, 323-337.

Lora, J. M., Lunine, J. I., Russell, J. L., Apr. 2015. GCM simulations of Titan's middle and lower atmosphere and comparison to observations. Icarus 250 , 516-528.

Lora, J. M., Mitchell, J. L., Aug. 2015. Titan's asymmetric lake distribution mediated by methane transport due to atmospheric eddies. Geophys. Res. Lett. 42, 6213-6220.

Lorenz, R. D., Mitchell, K. L., Kirk, R. L., Hayes, A. G., Aharonson, O., Zebker, H. A., Paillou, P., Radebaugh, J., Lunine, J. I., Janssen, M. A., Wall, S. D., Lopes, R. M., Stiles, B., Ostro, S., Mitri, G., Stofan, E. R., Jan. 2008. Titan's inventory of organic surface materials. Geophys. Res. Lett. 35, 2206.

Martin, E. C., Fitzgerald, M. P., McLean, I. S., Adkins, S. M., Aliado, T., Brims, G., Johnson, C., Magnone, K., Wang, E., Weiss, J., Aug. 2014. Performance modeling of an upgraded NIRSPEC on Keck. In: Ground-based and Airborne Instrumentation for Astronomy V. Vol. 9147 of Proc. SPIE . p. 914781.

McLean, I. S., Becklin, E. E., Bendiksen, O., Brims, G., Canfield, J., Figer, D. F., Graham, J. R., Hare, J., Lacayanga, F., Larkin, J. E., Larson, S. B., Levenson, N., Magnone, N., Teplitz, H., Wong, W., Aug. 1998. Design and development of NIRSPEC: a near-infrared echelle spectrograph for the Keck II telescope. In: Fowler, A. M. (Ed.), Infrared Astronomical Instrumentation. Vol. 3354 of Society of Photo-Optical Instrumentation Engineers (SPIE) Conference Series. pp. 566-578.

Mitchell, J. L., Aug. 2008. The drying of Titan's dunes: Titan's methane hydrology and its impact on atmospheric circulation. Journal of Geophysical Research (Planets) 113, E08015.

Mitchell, J. L., Sep. 2012. Titan's Transport-driven Methane Cycle. ApJ 756, L26.

Mitchell, J. L., Ádámkovics, M., Caballero, R., Turtle, E. P., Sep. 2011. Locally enhanced precipitation organized by planetary-scale waves on Titan. Nature Geoscience 4, 589-592.

Mitchell, J. L., Lora, J. M., 2016. Titan climate modeling. Annual Review of Earth and Planetary Sciences 44 (1).

Mitchell, J. L., Pierrehumbert, R. T., Frierson, D. M. W., Caballero, R., Sep. 2009. The impact of methane thermodynamics on seasonal convection and circulation in a model Titan atmosphere. Icarus 203, 250-264.

Montmessin, F., Fouchet, T., Forget, F., Mar. 2005. Modeling the annual cycle of HDO in the Martian atmosphere. Journal of Geophysical Research (Planets) 110, E03006.

Niemann, H. B., Atreya, S. K., Demick, J. E., Gautier, D., Haberman, J. A., 
Harpold, D. N., Kasprzak, W. T., Lunine, J. I., Owen, T. C., Raulin, F., Dec. 2010. Composition of Titan's lower atmosphere and simple surface volatiles as measured by the Cassini-Huygens probe gas chromatograph mass spectrometer experiment. Journal of Geophysical Research (Planets) 115 (E14), 12006.

Nixon, C. A., Temelso, B., Vinatier, S., Teanby, N. A., Bézard, B., Achterberg, R. K., Mandt, K. E., Sherrill, C. D., Irwin, P. G. J., Jennings, D. E., Romani, P. N., Coustenis, A., Flasar, F. M., Apr. 2012. Isotopic Ratios in Titan's Methane: Measurements and Modeling. ApJ 749, 159.

Penteado, P. F., Griffith, C. A., Greathouse, T. K., de Bergh, C., Aug. 2005. Measurements of $\mathrm{CH}_{3} \mathrm{D}$ and $\mathrm{CH}_{4}$ in Titan from Infrared Spectroscopy. ApJ 629, L53-L56.

Penteado, P. F., Griffith, C. A., Tomasko, M. G., Engel, S., See, C., Doose, L., Baines, K. H., Brown, R. H., Buratti, B. J., Clark, R., Nicholson, P., Sotin, C., Mar. 2010. Latitudinal variations in Titan's methane and haze from Cassini VIMS observations. Icarus 206, 352-365.

Perron, J. T., Lamb, M. P., Koven, C. D., Fung, I. Y., Yager, E., Ádámkovics, M., Nov. 2006. Valley formation and methane precipitation rates on Titan. Journal of Geophysical Research (Planets) 111 (10), 11001-+.

Rey, M., Nikitin, A. V., Campargue, A., Kassi, S., Mondelain, D., Tyuterev, V. G., 2016. Ab initio variational predictions for understanding highly congested spectra: rovibrational assignment of 108 new methane sub-bands in the icosad range $(6280-7800 \mathrm{~cm}-1)$. Physical Chemistry Chemical Physics (Incorporating Faraday Transactions) 18, 176-189.

Roe, H. G., May 2012. Titan's Methane Weather. Annual Review of Earth and Planetary Sciences 40, 355-382.

Roe, H. G., de Pater, I., Macintosh, B. A., Gibbard, S. G., Max, C. E., McKay, C. P., May 2002. NOTE: Titan's Atmosphere in Late Southern Spring Observed with Adaptive Optics on the W. M. Keck II 10-Meter Telescope. Icarus 157, 254-258.

Rothman, L. S., Gordon, I. E., Babikov, Y., Barbe, A., Chris Benner, D., Bernath, P. F., Birk, M., Bizzocchi, L., Boudon, V., Brown, L. R., Campargue, A., Chance, K., Cohen, E. A., Coudert, L. H., Devi, V. M., Drouin, B. J., Fayt, A., Flaud, J.-M., Gamache, R. R., Harrison, J. J., Hartmann, J.-M., Hill, C., Hodges, J. T., Jacquemart, D., Jolly, A., Lamouroux, J., Le Roy, R. J., Li, G., Long, D. A., Lyulin, O. M., Mackie, C. J., Massie, S. T., Mikhailenko, S., Müller, H. S. P., Naumenko, O. V., Nikitin, A. V., Orphal, J., Perevalov, V., Perrin, A., Polovtseva, E. R., Richard, C., Smith, M. A. H., Starikova, E., Sung, K., Tashkun, S., Tennyson, J., Toon, G. C., Tyuterev, V. G., Wagner, G., Nov. 2013. The HITRAN2012 molecular spectroscopic database. J. Quant. Spec. Radiat. Transf. 130, 4-50.

Schaller, E. L., Brown, M. E., Roe, H. G., Bouchez, A. H., May 2006a. A large cloud outburst at Titan's south pole. Icarus 182, 224-229.

Schaller, E. L., Brown, M. E., Roe, H. G., Bouchez, A. H., Trujillo, C. A., Oct. 2006b. Dissipation of Titan's south polar clouds. Icarus 184, 517-523.

Skidmore, W., TMT International Science Development Teams, Science Advisory Committee, T., Dec. 2015. Thirty Meter Telescope Detailed Science Case: 2015. Research in Astronomy and Astrophysics 15, 1945.

Stamnes, K., Tsay, S.-C., Jayaweera, K., Wiscombe, W., 1988. Numerically stable algorithm for discrete-ordinate-method radiative transfer in multiple scattering and emitting layered media. Applied Optics 27, 2502-2509.

Stofan, E. R., Elachi, C., Lunine, J. I., Lorenz, R. D., Stiles, B., Mitchell, K. L., Ostro, S., Soderblom, L., Wood, C., Zebker, H., Wall, S., Janssen, M., Kirk, R., Lopes, R., Paganelli, F., Radebaugh, J., Wye, L., Anderson, Y., Allison, M., Boehmer, R., Callahan, P., Encrenaz, P., Flamini, E., Francescetti, G., Gim, Y., Hamilton, G., Hensley, S., Johnson, W. T. K., Kelleher, K., Muhleman, D., Paillou, P., Picardi, G., Posa, F., Roth, L., Seu, R., Shaffer, S., Vetrella, S., West, R., Jan. 2007. The lakes of Titan. Nature 445, 61-64.

Tamai, R., Spyromilio, J., Jul. 2014. European Extremely Large Telescope: progress report. In: Ground-based and Airborne Telescopes V. Vol. 9145 of Proc. SPIE . p. 91451E.

Tokano, T., McKay, C. P., Neubauer, F. M., Atreya, S. K., Ferri, F., Fulchignoni, M., Niemann, H. B., Jul. 2006. Methane drizzle on Titan. Nature 442, 432435 .

Turtle, E., Del Genio, A., Barbara, J., Perry, J., Schaller, E., McEwen, A., West, R., Ray, T., 2011. Seasonal changes in titan's meteorology. Geophysical Research Letters 38 (3).

Turtle, E. P., Perry, J. E., Hayes, A. G., Lorenz, R. D., Barnes, J. W., McEwen, A. S., West, R. A., Del Genio, A. D., Barbara, J. M., Lunine, J. I., Schaller, E. L., Ray, T. L., Lopes, R. M. C., Stofan, E. R., Mar. 2011. Rapid and Extensive Surface Changes Near Titan's Equator: Evidence of April Showers.
Science 331, 1414-.

Turtle, E. P., Perry, J. E., McEwen, A. S., Del Genio, A. D., Barbara, J., West, R. A., Dawson, D. D., Porco, C. C., Jan. 2009. Cassini imaging of Titan's high-latitude lakes, clouds, and south-polar surface changes. Geophys. Res. Lett. 36 (2), L02204.

Villanueva, G. L., Mumma, M. J., Novak, R. E., Käufl, H. U., Hartogh, P., Encrenaz, T., Tokunaga, A., Khayat, A., Smith, M. D., Apr. 2015. Strong water isotopic anomalies in the martian atmosphere: Probing current and ancient reservoirs. Science 348, 218-221.

Xi, X., 2014. Review of Water Isotopes in Atmospheric General Circulation Models: Recent Advances and Future Prospects. International Journal of Atmospheric Sciences 2014 (250920), 16. 